\title{
PRIMARY LIPOSARCOMA OF THE LUMBAR SPINE: CASE REPORT
}

Frederico Barra de Moraes', André Luiz Passos Cardoso', Newłon Antônio Tristão', Wilson Eloy Pimenta Júnior², Sérgio Daher ${ }^{3}$, Siderley de Souza Carneiro ${ }^{4}$, Nathalia Parrode Machado Barbosa ${ }^{5}$, Nayanne de Lima Malta ${ }^{5}$, Noara Barros Ribeiro ${ }^{5}$

\section{ABSTRACT}

We report a rare case of primary bone liposarcoma of the lumbar spine, for which only one case has been reported. A female patient, 60 years of age, with lumbar pain and left sciatalgy for six months. In the imaging exams, a destructive tumor was found in the L4 vertebral body, and magnetic resonance imaging (MRI) revealed a tumoral lesion with T1 hiposignal and T2 hypersignal. Histological diagnosis was difficult, and immunohistochemistry confirmed the diagnosis. Surgical treatment was performed with wide ressection, spinal cord decompression, and anterior and posterior fusion of L3 to L5 complemented by radiotherapy and chemotherapy. After three years, a computed tomography (CT) scan evidenced an expansive injury in the lung. Despite its rarity, liposarcoma should be considered in the differential diagnosis of sciatica and primary tumors of the spine.

Keywords - Primary Liposarcoma; Bone Neoplasms; Lumbar Vertebrae

\section{INTRODUCTION}

Liposarcoma is a malignant soft-tissue tumor that originates from primitive mesenchymal cells, rather than from mature adipose tissue. There are five histological types, of which the pleomorphic type is the rarest. Pleomorphic liposarcoma is a high-grade tumor with highest prevalence between the ages of 60 and 70 years, and it affects both sexes equally. The commonest site for this tumor is the thigh, but other anatomical sites such as the lungs, heart, orbit, retroperitoneum, epidural space and bones ${ }^{(1)}$.

The first reports on primary liposarcoma of bone were made by Ewing, Stewart and Barnard in the 1930s, and Ewing included it in his classification of bone tumors in $1939^{(2-4)}$. However, its bone origin was subsequently questioned by several authors for some years. Among these was Jaffe, who only in the 1960s became convinced that it existed ${ }^{(5)}$. It is a very rare type of tumor, with less than 100 cases so far reported in the medical literature $^{(6)}$. Among these, primary pleomorphic liposarcoma of vertebrae is an even rarer lesion, with only two cases described (one in the thoracic spine and one in the lumbar spine).

The aim of this study was to report a case of primary liposarcoma of bone, in the lumbar spine, with descriptions of its clinical, radiological and treatment characteristics.

\section{CASE REPORT}

The patient was a 60 -year-old woman who started to present a condition of lumbalgia six months earlier. Initially, it improved using anti-inflammatory agents, but subsequently it evolved to left-side lumbar sciatic pain, with a positive Lasègue test at $40^{\circ}$, with progressive pain on the posterolateral face of the left lower limb, without any sensory or motor neurological deficit, and with symmetrical reflexes. Malignant fibrous histiocytoma and metastasis were suspected.

\footnotetext{
1 - Orthopedic Doctor of the Spine Service, Hospital das Clínicas of the Universidade Federal de Goiás do HC/UFG - Goiânia, GO, Brazil.

2 - Neurosurgeon of the Spine Service, HC/UFG - Goiânia, GO, Brazil,

3 - Head of the Spine Service of the HC- UFG and Head of the Orthopedics and Traumatology Department of the HC/UFG - Goiânia, GO, Brazil,

4 - Neurosurgeon, Spine Service of the HC/UFG - Goiânia, GO, Brazil,

5 - Medical Students of the Faculty of Medicine, HC/UFG - Goiânia, GO, Brazil,

Work carried out at the Orthopedics and Traumatology Department of the HC/UFG - Goiannia, GO.
}

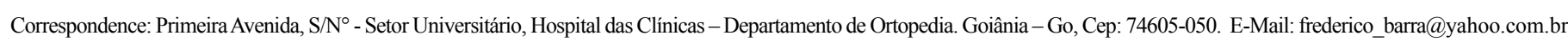
Received for publication: 01/26/2011, accepted for publication: 06/03/2011

The authors declare that there was no conflict of interest in conducting this work 
The initial radiographs on the lumbar spine did not show bone lesions (Figure 1). Computed tomography showed an extensive lytic lesion in the L4 body, in association with content presenting soft-tissue density. This was seen to be invading the medullary canal and neural foramens and compromising the nerve roots, especially on the left side (Figure 2). Nuclear magnetic resonance showed that the bone structure of the L4 body had been replaced by tissue presenting hyposignal in T1 and hypersignal in T2, with strong highlighting after introducing gadolinium, accompanied by invasion and compression of the medullary canal, without changes to the soft tissues (Figure 3).

A tomography-guided biopsy was then performed, from which an initial diagnosis of high-grade sarcomatous pleomorphic neoplasia was made (Figure 4). It was consequently decided to completely resect the L4 vertebra, initially using an anterior route. The body was replaced by a titanium cage filled with autologous bone graft harvested from the iliac, in order to obtain arthrodesis from L3 to L5.

Ten days later, in a second surgical procedure, the resection of L4 was completed by removing its posterior bone elements and decompressing the medulla, as well as finishing the arthrodesis from L3 to L5 with pedicled screws and bone graft material harvested from the iliac (Figure 5).

The histological evaluation showed that the bone tissue had been infiltrated by a pleomorphic malignant neoplastic lesion with rounded elongated hyperchromatic nuclei, mitoses and poorly defined cytoplasm, and with a rich vascular network, areas of hemorrhage and multivacuolated lipoblasts compatible with highgrade liposarcoma. Immunohistochemical analysis was then performed with the following results: vimentin +, ENE +, CD31 -, CD34 -, JJF35 - (these last three were $\mathrm{CI}+$ ), AE1 -, AE3 -, EMA -, S100 - and desmin -, thus confirming the diagnosis of primary liposarcoma of bone (Figure 6).

To complement the oncological treatment, radiotherapy and chemotherapy were also administered. The patient used a Putti vest for six months and, 18 months after the operation, she was free from pain, without neurological deficit, without recurrences of the neoplastic lesion and without metastases. However, three years after the operation, computed tomography showed an expansive lesion in a lung, with destruction of the adjacent ribs and extension to the dorsal muscle layer.

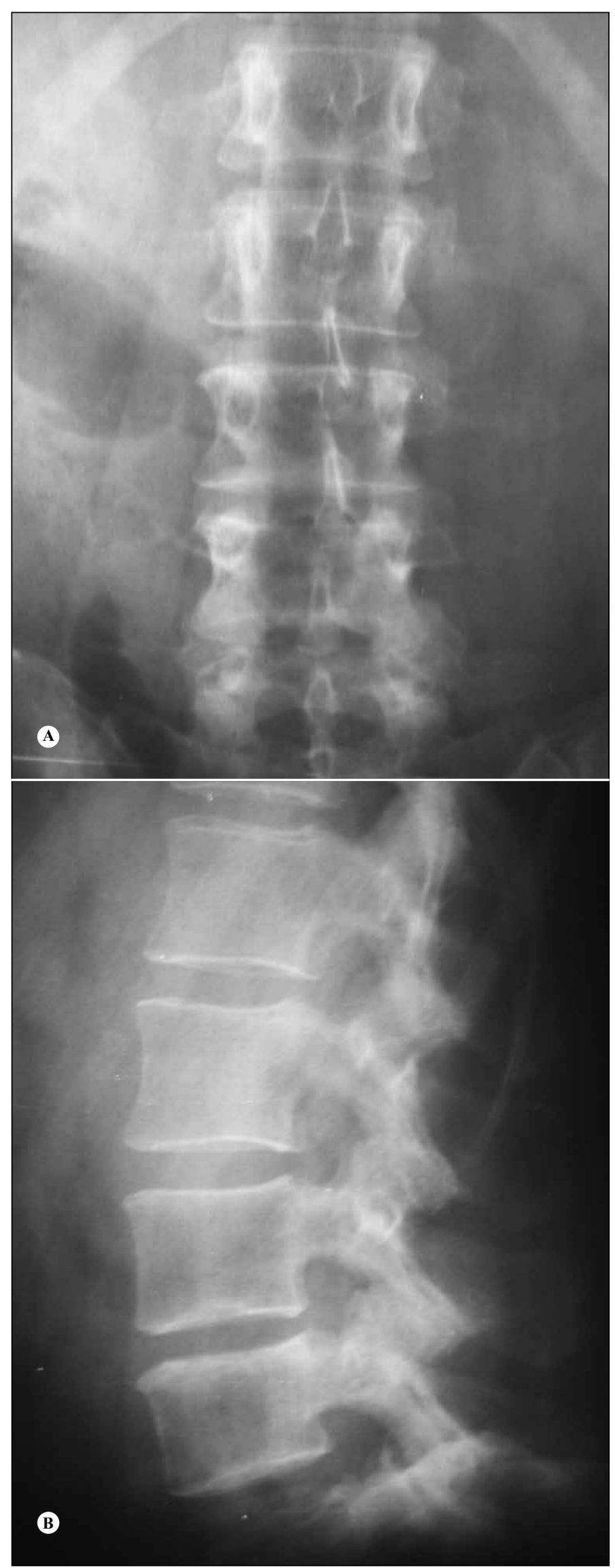

Figure 1 - Radiograph of the lumbar spine without evidence of the tumor at L4: anteroposterior view $(A)$ and lateral view (B). 


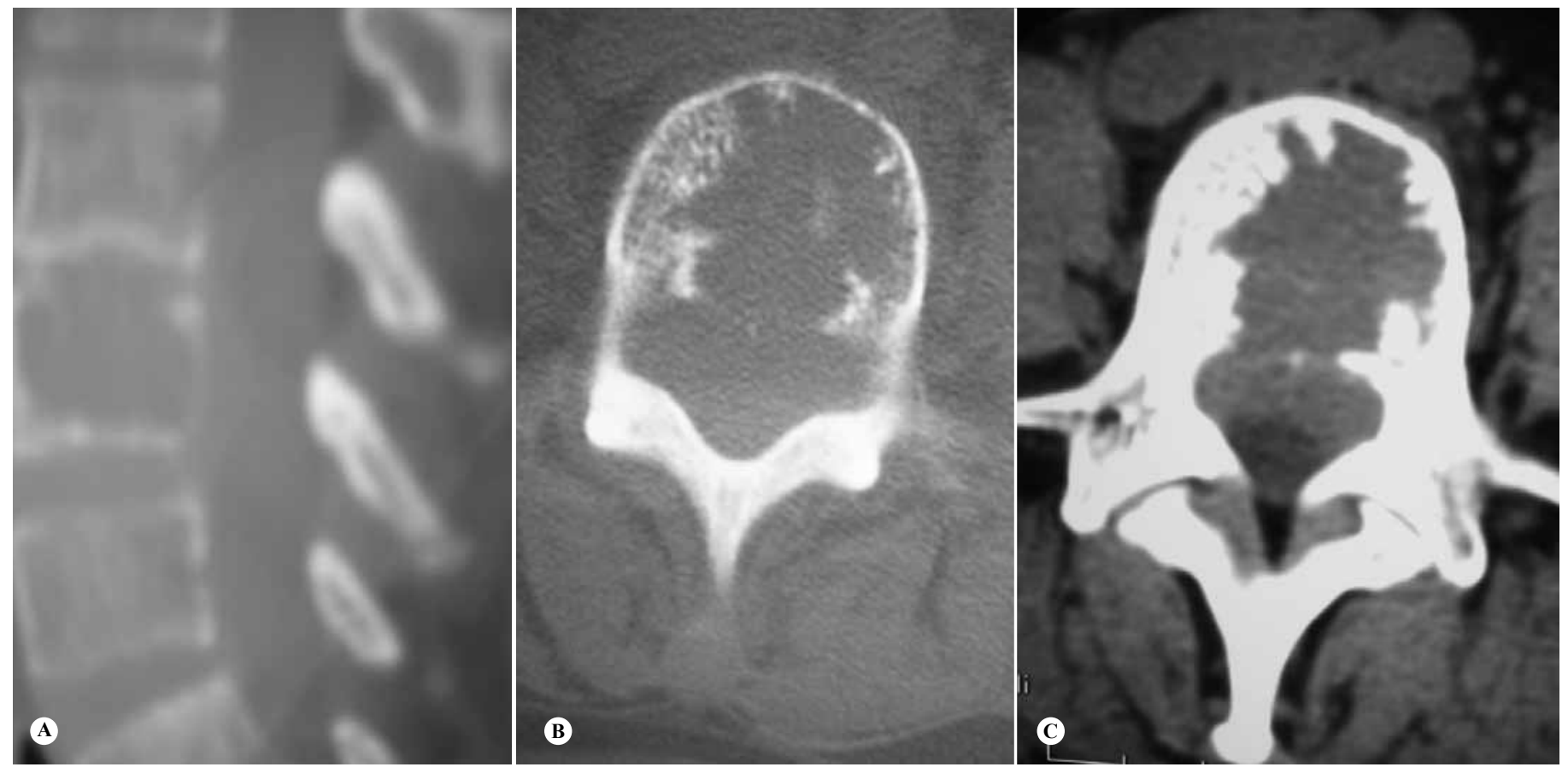

Figure 2 - Computed tomography of the lumbar spine, showing osteolytic lesion at L4, in sagittal slice (A). Axial slices: showing the destruction in a slice for bone (B) and tumor invasion of the canal in a slice for soft tissues (C).

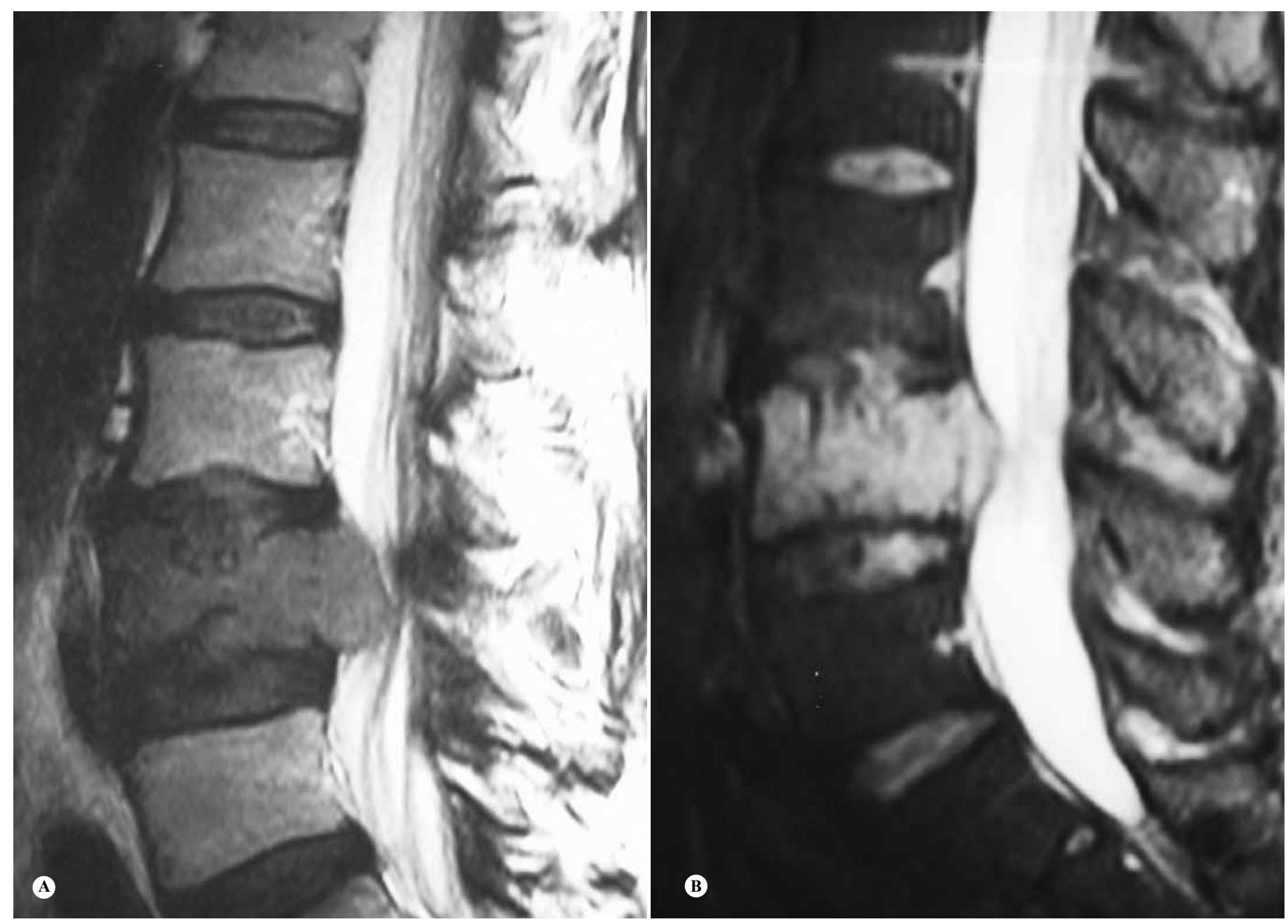

Figure 3 - Nuclear magnetic resonance imaging on the lumbar column, in a sagittal slice showing hyposignal in T1 (A) and hypersignal in T2 (B). Rev Bras Ortop. 2012;47(1):124-29 


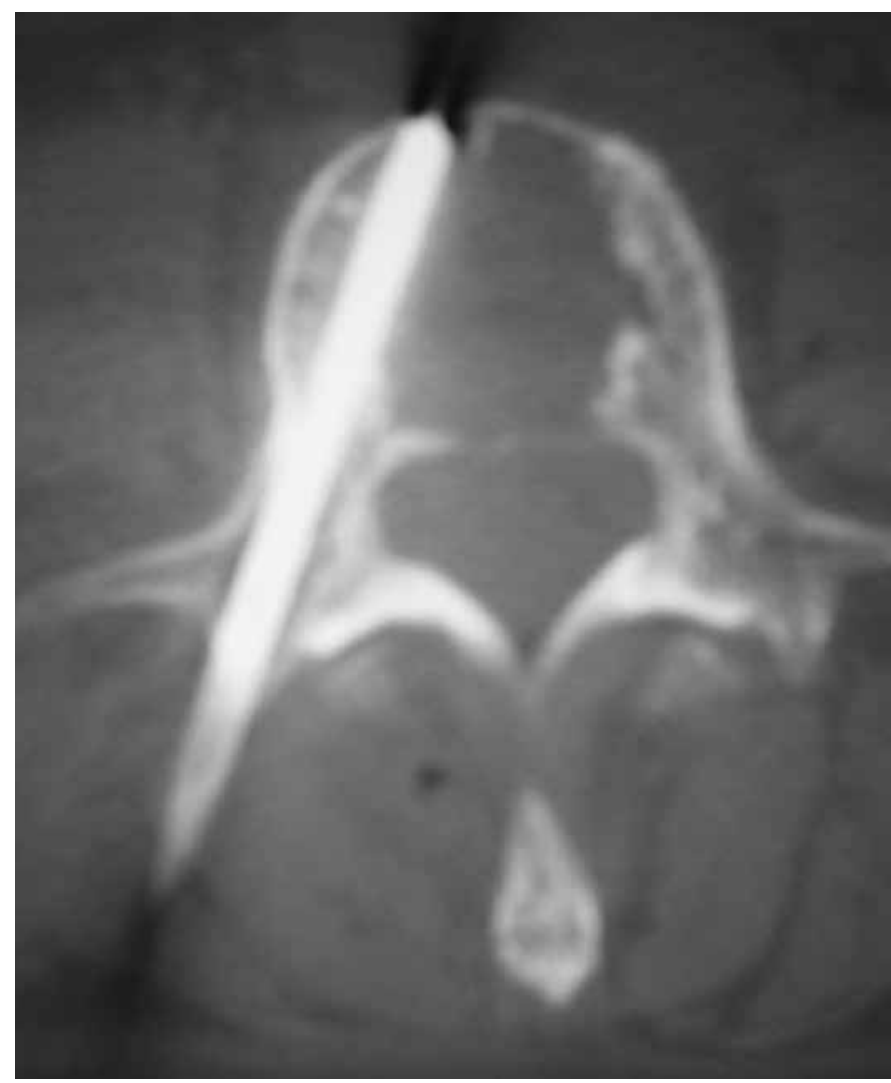

Figure 4 - Percutaneous biopsy using needle guided by computed tomography, in axial slice.

\section{DISCUSSION}

Lumbalgia is one of the commonest complaints at visits to primary-care physicians, and musculoskeletal complaints are commonest at emergency services ${ }^{(7)}$.

Lumbalgia is a common condition, responsible for significant morbidity, and it has an important economic and occupational impact within society ${ }^{(8)}$. Most patients with acute lumbalgia, with or without radicular symptoms, present musculoskeletal disorders or degenerative diseases that do not require specific treatment and are generally self-limited ${ }^{(9)}$. Physicians need to be alert to clinical indicators or "red flags" that suggest that systemic disease is present or neurological impairment is imminent. If there are no such findings, imaging diagnosis generally does not contribute towards case management and can be safely postponed. Continued activity among patients is associated with favorable results. Non-steroid anti-inflammatory drugs, paracetamol, muscle relaxants and tricyclic antidepressants may provide significant pain relief, and non-pharmacological measures may also contribute towards clinical and functional improvement ${ }^{(8)}$. However, the possibility that abnormalities
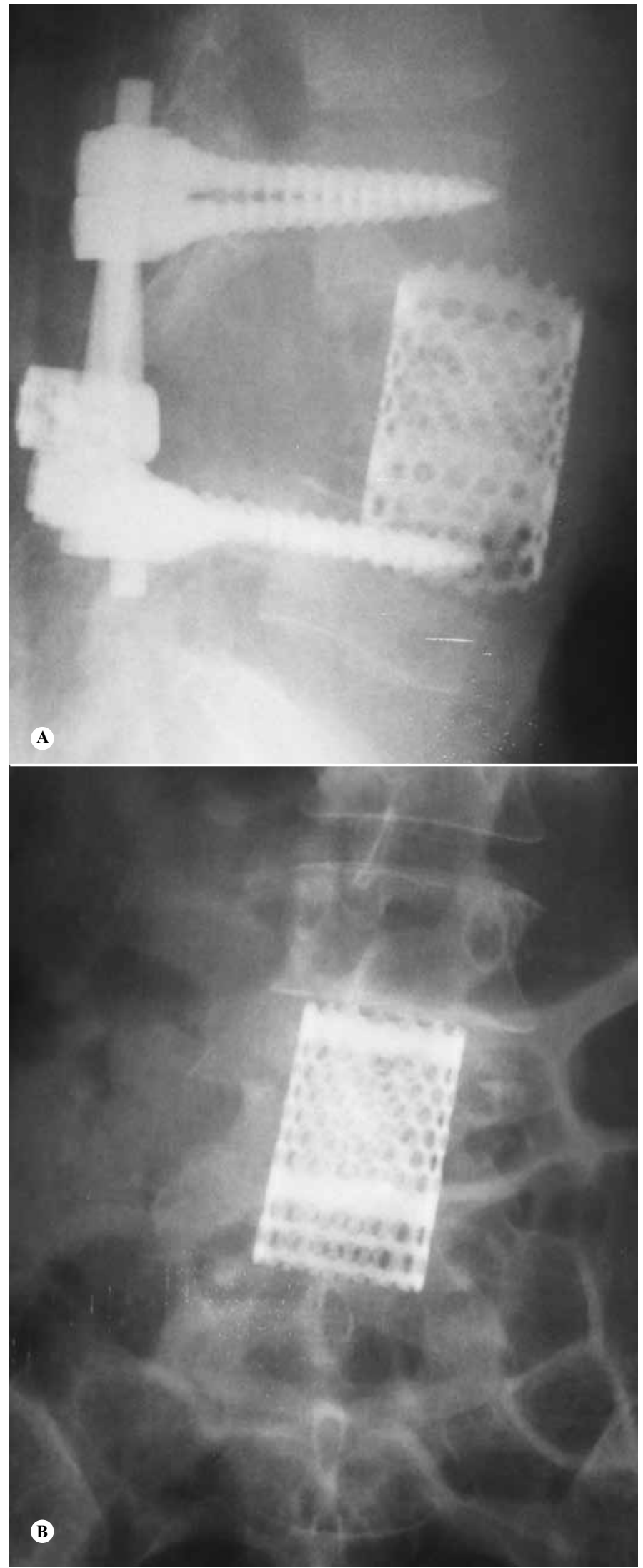

Figure 5 - Postoperative radiographs on lumbar spine showing resection of the L4 vertebral body and anterior arthrodesis with a titanium cage, in anteroposterior view (A), and medullary decompression and posterior arthrodesis with pedicled screws, in lateral view (B). 


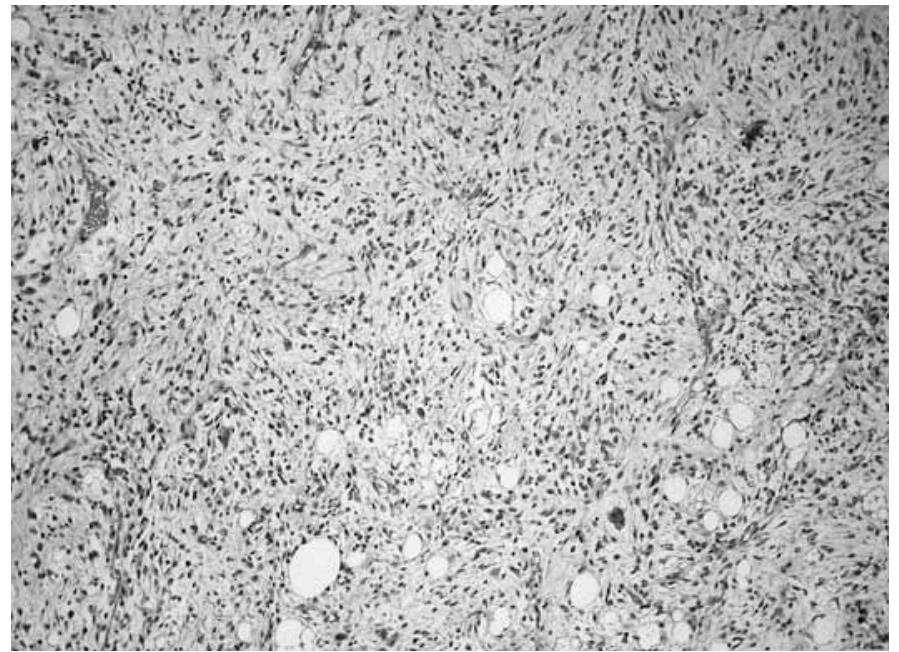

Figure 6 - Lesion showing high cellularity and cell atypia, compatible with fusocellular sarcoma, corresponding to the undifferentiated area (hematoxylin-eosin: 100x).

of greater severity may be present, requiring specific treatment, always needs to be ruled out ${ }^{(9)}$.

Most cases of lumbar pain are caused by mechanical conditions, including lumbar contraction or relaxation, degenerative disc and facet processes, disc hernia, vertebral canal stenosis and fracture compression due to osteoporosis. Non-mechanical conditions such as neoplasia, infections and inflammatory arthritis are important, but rarely cause lumbar pain. Furthermore, around $85 \%$ of evaluations on patients with lumbar pain do not reach a definitive diagnosis ${ }^{(9)}$.

Primary tumors of the spine are relatively uncommon types of neoplasia, in comparison with metastatic lesions, multiple myeloma and lymphoma. Their presentation is variable and insidious, before signs of spinal cord compression are detected, i.e. the point at which the diagnosis becomes evident ${ }^{(1)}$, as in the present case.

Primary liposarcoma of bone is preferentially found at the extremities of bones (tibia and femur). Three main morphological characteristics of pleomorphic liposarcoma have been described: high-grade MFH-like pleomorphic sarcoma, fusiform cell neoplasia and neoplasia with epithelioid morphology. Histologically, it can be divided into low, medium or high grade. It is formed by areas of normal, multivacuolated or even pleomorphic lipoblasts, with large quantities of mitoses. Lesions with low degrees of differentiation are highly vascular ${ }^{(1,10,11)}$.

The difficulty in diagnosing pleomorphic liposarcoma derives from its varied histological appearance. The histological criterion for diagnosing this neopla- sia consists of recognizing multivacuolated lipoblasts in a high-grade pleomorphic sarcoma. However, although the presence of lipoblasts is characteristic of any type of malignant tumor of adipocytes, this is insufficient for diagnosing liposarcoma, because lipoblasts also occur in a series of benign lesions. Liposarcoma can be confounded with malignant fibrous histiocytoma and malignant bone mesenchymoma, mainly because of the pleomorphic cell pattern ${ }^{(12)}$. It is difficult to differentiate pleomorphic liposarcoma from malignant fibrous histiocytoma. The latter is rarely seen in the spine and only nine cases have been described. Lipoblasts are not histological findings from this entity, and thus, this diagnosis was rejected in the present case. Immunohistochemical or ultrastructural analysis needs to be performed in order to establish the diagnosis ${ }^{(1)}$.

Although pleomorphic liposarcoma has a nonspecific immunohistochemical profile, special stains may be necessary in order to identify the presence of intracellular fat, rule out other substances such as mucin or glycogen and rule out other diagnoses. Like other sarcomas, pleomorphic liposarcoma expresses vimentin, while protein S100, smooth-muscle actin, cytokeratins AE1/AE3 and CAM5.2 have variable expression. Desmin is generally absent and the membrane antigen or CD34 is negative. The morphological characteristics and positivity for vimentin eliminate the hypothesis of blood diseases such as "signet ring" lymphoma or clear-cell myeloma ${ }^{(1,13)}$.

Radiographically, the lesions are destructive, osteolytic and poorly defined, and they are observed to expand and destroy the cortex. Nuclear magnetic resonance has great value for anatomical evaluations on spinal tumors, showing hyposignal in $\mathrm{T} 1$ and hypersignal in T2, mainly due to the non-lipomatous regions of the tumor. Since the radiological characteristics of spinal liposarcoma have only rarely been studied, no pathognomonic image has been described through any type of imaging in other parts of the body ${ }^{(1,10,11)}$.

Several factors are taken into account in planning the treatment, including location, degree of malignancy, tumor staging and presence or absence of metastasis. Wide or radical tumor resection should be performed, and local recurrences depend on this indication. Spinal instability caused by resection of the vertebral body and posterior elements should be 
avoided by means of vertebral arthrodesis ${ }^{(12)}$.

The radiosensitivity of primary liposarcoma of bone is greater than its radiocurability, and thus, radiotherapy is used as adjuvant and/or neoadjuvant treatment to diminish the size of the tumor. Primary liposarcoma of bone does not respond well to chemotherapy regimens, but these can be tried as adjuvant therapy for reducing the size of the tumor ${ }^{(3,4)}$.

Occurrences of metastases from liposarcoma have been reported in 40.2 to $45.8 \%$ of the cases in the literature. These cases often arose many years after apparently successful treatment of the primary tu- mor, and pulmonary metastases predominate. Thus, the prognosis for such cases would seem to be uncertain, with median survival of two years ${ }^{(1,6,14,15,16)}$.

Primary liposarcoma of the spine is a very rare disease, with only two cases reported in the literature. In cases of suspected primary tumors of the spine, pleomorphic liposarcoma should be considered in the differential diagnosis. Nuclear magnetic resonance has great value in investigating suspected spinal tumors, although the conclusive diagnosis of pleomorphic liposarcoma is only obtained through immunohistochemical and structural analysis.

\section{REFERENCES}

1. Hamlat A, Saikali S, Gueye EM, Le Strat A, Carsin-Nicol B, Brassier G. Primary liposarcoma of the thoracic spine: case report. Eur Spine J. 2005;14(6):613-8.

2. Ewing JA. A review of the classification of bone tumors. Bull Am Coll Surg 1939;24:290-5

3. Stewart FW. Primary Liposarcoma of Bone. Am J Pathol. 1931;7(2):87-94.5.

4. Barnard L. Primary liposarcoma of bone. Arch Surg. 1934;29:560-5.

5. Jaffe HL. Tumors and tumorous conditions of the bones and joints. Philadelphia: Lea \& Febiger; 1958.

6. Torok G, Meller Y, Maor E. Primary liposarcoma of bone. Case report and review of the literature. Bull Hosp Jt Dis Orthop Inst. 1983;43(1):28-37.

7. Corwell BN. The emergency department evaluation, management, and treatment of back pain. Emerg Med Clin North Am. 2010;28(4):811-39.

8. Duffy RL. Low back pain: an approach to diagnosis and management. Prim Care. 2010;37(4):729-41.

9. Goldman L, Ausiello D. Cecil tratado de medicina interna. Rio de Janeiro: Elsevier; p. 2609-12.

10. Campanacci M. Bone and soft tissue tumors. New York: Springer; 1990.
11. Schajowicz F. Neoplasias ósseas e lesões pseudotumorais. Rio de Janeiro: Revinter; 2000.

12. Simmon M, Springfield D. Surgery for bone and soft tissue tumors. Philadelphia: Lippincott-Raven Publishers; 1997.

13. Oliveira AM, Nascimento AG. Pleomorphic liposarcoma. Semin Diagn Pathol. 2001;18(4):274-85.

14. Addison AK, Payne SR. Primary liposarcoma of bone. Case report. J Bone Joint Surg Am. 1982;64(2):301-4.

15. Schneider HM, Wunderlich T, Puls P. The primary liposarcoma of the bone. Arch Orthop Trauma Surg. 1980;96(3):235-9.

16. Ishii T, Ueda T, Myoui A, Tamai N, Hosono N, Yoshikawa H. Unusual skeletal metastases from myxoid liposarcoma only detectable by MR imaging. Eur Radiol. 2003;13(Suppl 4):L185-91. 